\title{
Les Arts libéraux dans la tapisserie à la fin du Moyen Âge : entre iconographie savante et pratiques d'atelier.
}

\section{Pascale CHARRON, Université de Tours-CESR/UMR 7323}

Si Martianus Capella, dans ses Noces de Philologie et de Mercure composées durant la première moitié du $\mathrm{V}^{\mathrm{e}}$ siècle, n'est pas à l'origine de l'organisation du savoir en une série de disciplines, il en est sans nul doute le promoteur le plus célèbre et le plus important ${ }^{1}$. En choisissant l'allégorie pour donner corps aux disciplinae que Mercure offre comme servantes à son épouse au moment de leurs noces, il assure à son texte une notoriété qui sera particulièrement forte au cours de la période carolingienne ${ }^{2}$. Si les deux premiers livres sont consacrés à la narration du mariage du dieu Mercure avec la Philologie, transportée à cette occasion dans les cieux jusqu'au Mont Olympe, les sept livres suivant sont dédiés aux arts libéraux et s'organisent autour d'une description physique des allégories féminines comme de leurs attributs et d'un exposé sur l'histoire et les fondamentaux des différentes sciences. Défilent ainsi, sous les yeux des dieux assemblés pour les noces, la Grammaire, la Dialectique, la Rhétorique, la Géométrie, l'Arithmétique, l'Astronomie et la Musique. Les nombreux commentaires de l'œuvre de Martianus, rédigées au cours de la période carolingienne, témoignent de l'attraction exercée par ce texte sur le monde intellectuel chrétien. Constituant les sept colonnes de la Sagesse divine pour Alcuin ${ }^{3}$ les arts libéraux sont

\footnotetext{
${ }^{1}$ « Reste que Martianus, s'il n'est pas le pilier du système médiéval du triuium et du quadriuium, en constitue évidemment l'un des piliers : son influence se marque particulièrement par la fortune des allégories qu'il a imaginées pour décrire chacune des uirgines/disciplinae [...]», Martianus Capella, Les Noces de Philologie et de Mercure, Tome VII, Livre VII, L’Arithmétique, J.-Y. GUILLAUMIN (tr.), Paris, 2003, p. XIX. Sur les origines de l'organisation encyclopédique du savoir dans le monde antique, idem, p. XLVII-LVI.

${ }^{2}$ M.-Th. D'ALVERNY, «La sagesse et ses sept filles. Recherches sur les allégories de la Philosophie et des Arts libéraux du $\mathrm{IX}^{\mathrm{e}}$ au XII ${ }^{\mathrm{e}}$ siècle », dans Mélanges dédiés à la mémoire de Félix Grat, Paris, 1946, vol. 1, p. 245-278.

${ }^{3}$ Prologue du De grammatica, P.L., CI, 853. Cité par D’ALVERNY, p. 245.
} 
ainsi compris, à la suite de Cassiodore qui encourage ses lecteurs «à lire l'exposé des sept sciences $»^{4}$, comme une propédeutique permettant l'accès aux Ecritures.

Constituant ce que les penseurs carolingiens nomment, à la suite de Boèce, le quadrivium (géométrie, arithmétique, astronomie et musique) et de ce qu'ils baptisent désormais trivium (grammaire, dialectique et rhétorique), les arts libéraux semblent avoir été un sujet de représentation dès le $\mathrm{IX}^{\mathrm{e}}$ siècle. Les rares images qui en ont été conservées se résument principalement à quelques dessins et enluminures ${ }^{5}$ et à des descriptions poétiques de décors peints attestant de leur présence dans certains édifices ${ }^{6}$. La mise en place d'une véritable tradition iconographique s'opère seulement à partir du $\mathrm{XII}^{\mathrm{e}}$ siècle ${ }^{7}$. Les sculptures des voussures de la porte nord du Portail Royal de la cathédrale de Chartres $^{8}$ en sont l'exemple majeur qui impose une formule iconographique demeurant pérenne jusqu'à la fin du Moyen Âge. Les arts sont représentés trônant sous leur forme féminine tenant des attributs et étant accompagnées, sur les claveaux qui les précèdent, de leurs acteurs antiques. Bien qu'aucune liste canonique n'en ait jamais été dressée, la tradition picturale a imposé une suite restreinte de savants et de philosophes associés à chaque art. Ainsi la Grammaire est-elle accompagnée de Donat ou de Priscien, la Dialectique d'Aristote, la Rhétorique de Tullius ou de Cicéron, la Géométrie et l'Arithmétique d'Euclide ou de Pythagore, l'Astronomie de Ptolémée et la Musique de Tubalcaïn. Il en est de même pour leurs attributs qui sont rarement

\footnotetext{
${ }^{4}$ Cassiodore (v. 490-v. 580), qui n'a pas lu le De nuptiis de Marcianus Capella, introduit cependant dans ses Institutiones les sept arts libéraux, GUILLAUMIN, op. cit. (notre note 1) p. XXIX.

${ }^{5} \mathrm{Ph}$. VERDIER, «L'iconographie des Arts libéraux dans l'art du Moyen Âge jusqu'à la fin du quinzième siècle », dans Arts libéraux et Philosophie au Moyen Âge, Actes du $4^{\text {ème }}$ congrès international de Philosophie au Moyen Âge, Montréal, 1969, p. 307-308

${ }^{6}$ Théodulfe, évêque d'Orléans, Poème De septem liberalibus artibus in quadam pictura depictis, cf. D'ALVERNY, op. cit. (notre note 2), p. 255. M. EVANS, («Allegorical women and practical men : the iconography of the artes reconsidered », dans D. BAKER (éd.), Medieval Women, Oxford, 1978, p. 305-307) considère que les textes lus par la tradition comme des descriptions d'œuvres sont avant tout des œuvres littéraires ou poétiques qui ne renvoient pas à une réalité picturale.

7 A. KATZANELLENBOGEN, "The Representation of the Seven Liberal Arts », dans Twelfthcentury Europe and the foundations of modern Society, Université du Wisconsin, Madison/Wis., 1961, p. 39-55.

${ }^{8}$ La présence des arts libéraux sur le portail de Chartres est en lien avec l'école de Chartres au sein de laquelle les arts libéraux tiennent une place considérable: M. ZINK, G. HASENHOR (dir.), Dictionnaire des Lettres Françaises. Le Moyen Âge, Paris, 1992, p. 255-257 (bibliographie).
} 
en accord avec les portraits le plus souvent complexes que Martianus Capella dresse des servantes de la Philologie. Celui-ci en faisant œuvre littéraire ne conçoit pas ses descriptions comme étant destinées à une traduction picturale. C'est en grande partie pour cette raison que l'aspect physique, les vêtements et les attributs des figures féminines ne répondent que rarement à la description initiale ou du moins en présente une version simplifiée, voire parfois détournée par les traductions successives. Si le temps nous manque dans le cadre de ce colloque pour rappeler la liste de ces variantes, qui ont été par ailleurs l'objet d'études antérieures $^{9}$, il est important de considérer le fait que la formule iconographique stabilisée à défaut d'être inventée à Chartres demeure celle qui s'impose majoritairement sous ses aspects génériques durant tout le Moyen Âge.

Dans le corpus des nombreuses représentations des arts libéraux, la tapisserie ne tient pas une bien grande place en terme quantitatif, puisque seules six pièces sont aujourd'hui conservées $^{10}$, et ne l'a jamais tenue si l'on en croit les inventaires de biens mobiliers et les comptes qui nous sont parvenus. L'inventaire des biens de Charles V dressé en 1380 mentionne ainsi trois tapisseries représentant les arts libéraux : «Item, un grand beau tappiz que le roy a acheté, qui est à ouvraige d'or, ystorié des sept sciences et de saint Augustin »; «Item, le tappis des Sept sciences, qui fut à la royne Jehanne d'Evreux »; «Item, ung autre

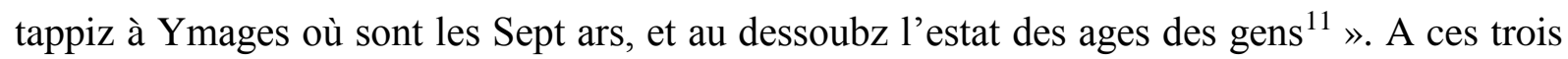
mentions s'ajoute celle d'un paiement de Philippe le Hardi (1384) : «pour un drap de haulte lice achaté par Mgr audit Jehan Cosset la somme de cent frans d'or, lequel drap est de

\footnotetext{
${ }^{9}$ VERDIER, op. cit. (notre note 5), p. 312-322.

${ }^{10}$ L'Arithmétique et l'Astronomie, Memorial Art Gallery, Rochester; La Géométrie, Museum of Freemasonry, Londres; La Rhétorique, Musée des Arts Décoratifs, Paris ; L'Arithmétique, Musée National du Moyen Âge, Paris ; La Musique, Museum of Fine Arts, Boston ; L'Astronomie, Musée de Göteborg.

${ }^{11}$ J. LABARTE, Inventaire du mobilier de Charles V, roi de France, Paris, 1879, p. 379, articles 3691, 3692, 3699.
} 
l'histoire des sept ars de sciences $^{12}{ }^{2}$. La liste s'arrête là. En l'état actuel de nos recherches, nous ne connaissons pas d'autre mention de tentures représentant ce thème pour la période médiévale ${ }^{13}$. Les quatre pièces décrites, qui datent de la seconde moitié du $\mathrm{XIV}^{\mathrm{e}}$ siècle, correspondent au début de l'essor de la tapisserie dans le royaume de France et sur les terres bourguignonnes. Ces tapisseries présentent les arts libéraux rassemblés sur une seule pièce et par deux fois associés à un autre thème. Saint Augustin trouve naturellement sa place auprès du trivium et du quadrivium en tant que promoteur chrétien de la hiérarchisation du savoir ${ }^{14}$. Les sept âges de la vie sont par ailleurs une association inédite renvoyant certainement à la pratique encyclopédique du classement, les étapes de la vie humaine ici présentées suivant sept états faisant écho aux sept disciplines du savoir universitaire. Pour les tapisseries ayant appartenues à Jeanne d'Evreux ${ }^{15}$ et à Philippe le Hardi, le thème semble se suffire à luimême : sept arts ou sept sciences dont nous ignorons l'aspect en raison de la brièveté des items mais que nous pouvons supposer fidèle au prototype précédemment décrit.

Les tapisseries conservées correspondent chronologiquement à la fin de la période médiévale et ne répondent pas aux œuvres décrites dans ces inventaires du XIV ${ }^{\mathrm{e}}$ siècle. En effet, il ne s'agit plus là du regroupement des arts sur une tapisserie unique mais la représentation des personnifications des sciences isolées ou regroupées par deux. Les projets semblent dès lors plus ambitieux car ils entraînent la multiplication des pièces pour composer

\footnotetext{
${ }^{12} \mathrm{~B}$. et H. PROST, Inventaires mobiliers et extrait des comptes des ducs de Bourgogne et de la maison de Valois, Paris, 1902-1913, II, p. 166, $\mathrm{n}^{\circ}$ 1109. Jean Cosset est marchand, bourgeois d'Arras, valet de chambre du duc de Bourgogne, Philippe le Hardi.

${ }^{13} \mathrm{Au} \mathrm{XVII}$ siècle, le thème semble être plus en vogue comme en témoigne la tenture de huit pièces représentant les Arts libéraux et leur apothéose, selon des cartons de Cornelis Schut ; à ce sujet, G. DELMARCEL, E. DUVERGER, Bruges et la tapisserie, Bruges/Mouscron, 1987, p. 453-489.

${ }^{14}$ R. I. MARROU, «Les arts libéraux dans l'antiquité classique », dans Arts libéraux et Philosophie au Moyen Âge, Actes du $4^{\text {ème }}$ congrès international de Philosophie au Moyen Âge, Montréal, 1969, p. 5-27.

${ }^{15}$ Cette tapisserie décrite comme ayant appartenue à la reine Jeanne d'Evreux n'est pas mentionnée dans le «compte de l'exécution » de son testament daté du 28 septembre 1372 : C. LEBER, Collection des meilleurs dissertations, notices et traités particuliers relatifs à l'histoire de France composée, en grande partie, de pièces rares, ou qui n'ont jamais été publiées séparément, pour servir à compléter toutes les collections de mémoires sur cette matière, Paris, 1838, T. XIX, p. 120-169.
} 
une tenture complète si c'est bien ainsi que nous devons lire ces œuvres, c'est-à-dire comme les éléments d'un ensemble plus vaste mais désormais isolés en raison des hasards de la conservation. Cependant, il ne faut pas écarter l'hypothèse de tapisseries ne représentant qu'un seul art sorti dès lors de sa classification universitaire. Cette question renvoie à celle de la fonction première de ces œuvres. Si celle-ci nous échappe au premier abord en raison de l'absence de pièces d'archives permettant d'en identifier la commande, les circonstances et les lieux de production, les tentures cependant par les choix iconographiques qu'elles exposent mais également par les traces lisibles de leur mise en œuvre permettent d'éclaircir un peu cette question et de qualifier plus avant les spécificités de la représentation des arts libéraux en tapisserie.

Le premier ensemble est composé de deux pièces datées en raison de leur style autour de 1460 et répondant au même type de formule iconographique comme au même ensemble de cartons préparatoires. La tapisserie appartenant au Musée de Rochester montre l'Arithmétique et l'Astronomie, deux arts du quadrivium, identifiées par des inscriptions (Fig. 1). Dévoilées par les anges qui écartent les pans des pavillons sous lesquels elles sont représentées, elles trônent devant des tentures d'honneur. A leurs pieds sont assis deux hommes dans lesquels nous pouvons reconnaître les savants de l'antiquité désormais traditionnels, identifiés hypothétiquement ici comme étant Pythagore et Ptolémée ${ }^{16}$. Les arts sont munies d'attributs : main droite levée en signe d'autorité et dans la main gauche une tablette où sont reportés des chiffres arabes pour l'Arithmétique ; les yeux au ciel et tenant une sphère armillaire pour 1'Astronomie ${ }^{17}$. La tapisserie du Musée de la Franc-maçonnerie de Londres ${ }^{18}$ (Fig. 2) montre

\footnotetext{
${ }^{16}$ D. BURGER, «Old Tapestries representing the seven Liberal Arts », Archives Internationales d'Histoire des Sciences, t. XXIX, 1950, p. 870.

${ }^{17} \mathrm{Ce}$ dernier instrument renvoie directement à Ptolémée car il était réputé pour avoir été son outil de prédilection au cours de son observation des étoiles.

${ }^{18} \mathrm{G}$. BRETT, «Geometry, Ars Quatuor Coronatorum. Being the Transactions of the Quatuor Coronati Lodge, $\mathrm{n}^{\circ}$ 2076, 1955, p. 132-133. Cette tapisserie a été achetée par le Musée de la Francmaçonnerie de Londres lors de la vente Weinmüller des 11-13 mai 1955 à Munich. Je remercie M.
} 
l'allégorie de la Géométrie dans une posture identique, tenant un compas et une équerre et en même compagnie (Euclide $?^{19}$ ). Ce type de représentation renvoie directement à l'une des fresques de la salle capitulaire du couvent dominicain de Santa Maria Novella à Florence ${ }^{20}$ (aujourd'hui nommée Chapelle des Espagnols ${ }^{21}$ ) réalisée par Andrea Bonaiuto (di Firenze) entre 1365 et 1367. Cette œuvre (Fig. article de Daniel Russo) développe sur le mur occidental de la chapelle un grand ensemble consacré à la célébration de la gloire de saint Thomas d'Aquin ${ }^{22}$. Elle présente, disposées en pendant, les allégories des droits canons et civil, de la théologie et des sciences théologales et les disciplines universitaires. Toutes ces personnifications figurées en rang, sont placées sur des trônes dominés par des dais architecturés assez semblables aux stalles présentes dans les chœurs des édifices à la même époque. Faisant face aux spectateurs, dans ce cas la communauté dominicaine du couvent, elles ont à leurs pieds le philosophe ou le savant qui leur correspond. Si le peintre ne s'éloigne pas des modèles existant depuis le $\mathrm{XII}^{\mathrm{e}}$ siècle, il en propose une formule renouvelée en établissant une relation hiérarchique entre l'Art et son représentant. Ce schéma va connaître une fortune importante dans le siècle qui suit comme le montrent les peintures murales de la bibliothèque de la cathédrale du Puy (Fig. 3) ${ }^{23}$, les panneaux peints par Juste de Gand pour le

Martin Cherry, bibliothécaire de la Library and Museum of Freemasonry, pour sa grande disponibilité et pour les photographies gracieusement mises à notre disposition.

19 Ibid., p. 133.

${ }^{20}$ Cette chapelle qui ouvre sur le Chiostro verde, possède à l'origine une double fonction. Elle est tout d'abord la chapelle funéraire de Buonamico Guidalotti, riche marchand florentin qui lègue par testament (2 août 1335) une forte somme aux dominicains pour l'ornementation et la réalisation de peintures dans ce lieu et qui y fait placer son tombeau. Sa seconde fonction est celle de salle de réunion du chapitre de la communauté. Les peintures répondent à cette double fonction, à ce sujet se reporter dans ce même volume à l'article de Daniel RUSSO, et à D. NORMAN, «The art of Knowledge: two artistic schemes in Florence », dans NORMAN, D. (éd.), Florence, Siena and Padua. Art, society and religion 1280-1400, Vol. II, Case Studies, Londres, 1995, p. 217-241 et VERDIER, op. cit. (notre note 5) , p. 222-228.

${ }^{21}$ Vers 1556, la communauté espagnole de Florence obtiendra de la communauté dominicaine l'usage de cette chapelle pour ses dévotions privées.

${ }^{22}$ Le Triomphe de saint Thomas d'Aquin exalte ses fonctions de théologien et d'enseignant en insistant sur l'inspiration divine de ses œuvres, Norman, p. 227.

${ }^{23}$ A. MASSON, «Les arts libéraux du Puy et la décoration des bibliothèques à la fin du Moyen Âge », Comptes-rendus de l'Académie des inscriptions et belles-lettres, $\mathrm{n}^{\circ} 2$, 1958, p. 150-170; N. LEBLOND, «Le chanoine Pierre Odin, commanditaire de la peinture murale des Arts libéraux pour la 
studiolo de Gubbio ${ }^{24}$ ou les fresques de Pinturrichio pour les appartements Borgia au Vatican. Dans ces œuvres, les arts sont maintenus dans des postures frontales et surélevées par rapport à leurs compagnons. Si ceux-ci perdent leur immobilité primitive, ils confirment par leur posture la domination de la discipline dont ils sont les serviteurs. Les tapisseries de Rochester et de Londres répondent, elles aussi, à cette disposition des personnages, la hiérarchie entre les deux étant plus encore clairement établie par la plus petite taille donnée aux figures masculines.

La particularité des différents lieux conservant ou ayant reçus ces représentations est d'être consacrée au savoir et à sa célébration ${ }^{25}$. Si la salle du Chapitre des dominicains de Santa Maria Novella exalte les fonctions de théologien et d'enseignant de saint Thomas d'Aquin, elle célèbre par le fait les fonctions didactiques de la communauté toute entière rassemblée en ce lieu. Les peintures de la bibliothèque de la cathédrale du Puy-en-Velay, commandées par le chanoine Pierre Odin à la fin du $\mathrm{XV}^{\mathrm{e}}$ siècle, répondent certainement à la même ambition, celle de magnifier un lieu essentiel de la vie des chanoines comme de leur identité communautaire par la représentation des trivium et quadrivium associés. La salle des Arts libéraux du Vatican est identifiée comme ayant très certainement été la bibliothèque des appartements d'Alexandre VI Borgia ${ }^{26}$ et le studiolo de Frédéric de Montefeltre demeure l'un

bibliothèque capitulaire de la cathédrale du Puy », dans F. JOUBERT (dir.), L'artiste et le clerc, Paris, 2006, p. 371-388 ; R. VINER, "The Mural of the Liberal Arts in the Library of Le Puy Cathedral », dans Pictorial Languages and their Meanings : liber amicorum in honor of Nurith Kenaan-Kedar, Tel Aviv University, 2006, p. 265-272.

${ }^{24}$ O. RAGGIO, « The Liberal Arts Studiolo from the Ducal Palace at Gubbio », The Metropolitan Museum of Art Bulletin, vol. 53, $\mathrm{n}^{\circ} 4$ (printemps 1996), p. 3-35.

${ }^{25} \mathrm{~L}$ 'association des arts libéraux et des bibliothèques est connue pour d'autres sites et ceci jusqu'à la période moderne. A ce sujet se reporter à A. MASSON, «Les Arts libéraux du Puy et le décoration des bibliothèques à la fin du Moyen Âge ", Comptes-rendus des séances de l'Académie des Inscriptions et belles-lettres, $\mathrm{n}^{\circ} 2$ 2, 1958, p. 150-170; C. LAUTIER, «Les arts libéraux de la "librairie » capitulaire de Chartres », Gesta, XXXVII/2, Essays on Stained Glass in Memory of Jane Hayward (1918-1994), 1998, p. 211-216 ; R. MARKS, Stained Glass in England during the Middle Ages, Londres, 1993, p. 101 ; C. COPPENS, «Seven Liberal Arts and Ten Commandments : the decoration intended for the Library of the Celestine Monastery of Heverlee, Louvain », Quaerendo, $\mathrm{n}^{\circ}$ 28, 1998, p. 243-272.

${ }^{26}$ C. CIERI-VIA, N. BLAMOUTIER, "Characteres et figures in opera magico". Pinturrichio et la décoration de la «camera segreta » de l'appartement Borgia », Revue de l'art, 1991, n 1, p. 12. 
des lieux d'étude les plus exceptionnels de la Renaissance italienne à la fois par la complexité de son programme iconographique et par son décor d'intarsia illusionnistes et de panneaux peints aujourd'hui dispersés et en partie détruits ${ }^{27}$. Les tapisseries qui nous intéressent, dont la formulation iconographique est si proche des œuvres peintes observées, doivent être regardées comme participant de ce même courant de célébration d'un savoir considéré comme l'un des fondements de la pensée chrétienne. Les anges, par leur geste de révélation, comme les inscriptions latines encore lisibles sur la pièce de Rochester, mais en grande partie disparues sur la tapisserie de Londres, en témoignent également ${ }^{28}$. Le choix du latin est ici en adéquation parfaite avec les thèmes représentés et indique implicitement que l'œuvre s'adresse à un public lettré ${ }^{29}$.

La tapisserie de Londres qui répond en tous points aux critères énoncés introduit cependant une variante importante à l'orientation programmatique choisie. Il s'agit de la représentation de praticiens au coté du théoricien. Nous retrouvons là la distinction entre artifex theorice et artifex pratice qui traverse toute la période médiévale ${ }^{30}$. La géométrie est accompagnée d'ouvriers penchés sur un bloc de pierre tenant une équerre et un gabarit et,

\footnotetext{
${ }^{27}$ Les peintures furent retirées du studiolo dès 1673. Deux des panneaux représentant l'Astronomie et la Dialectique, conservés au Kaiser Friedrich Museum à Berlin furent détruites en 1945.

${ }^{28}$ L'inscription portée sur la pièce de Rochester demeure difficile à lire et a fait l'objet de nombreux commentaires : "R.P. Mesurans et[iam] coeli p[rae] /sumo figuras", à ce sujet se reporter à BURGER, op. cit. (notre note 15), p. 870-871. Sur l'œuvre de Londres, l'inscription est incomplète, BRETT (op. cit. notre note 17) propose d'y voir les mots « et sit proporcio rerum » sans certitude. Ces citations latines sont dans la tradition des sentences inscrites sur des phylactères sur les peintures murales du Puy-en-Velay. Grammaire : "Quicquid agant artes, ego simper praedico partes » (Quoi que traitent les arts, je suis celle qui toujours en avance les parties) ; Logique ou Dialectique : «Me sine, doctores frustra coluere sorores » (Sans moi, c'est en vain que les docteurs ont honorés mes soeurs); Rhétorique : «Est mihi dicendi ratio, cum flore loquendi » (La manière de dire m'appartient, ainsi que la fleur du langage ); Musique : «Invenere locum per me modulamina vocum» (La mesure des voix par moi a trouvé sa place), N. LEBLOND, op. cit. (notre note 22) p. 374.

${ }^{29}$ Sur la question de la langue utilisée dans les tituli des tapisseries se reporter à : M. GÉREZ, «Cartouches, phylactères, inscriptions libres : le texte dans les tapisseries de la fin du Moyen Âge », dans Ch. HECK (dir.), Lecture, représentation et citation. L'image comme texte et l'image comme signe (XIe-XVIIe siècle), Lille, 2007, p. 158-159.

30 «Il [le Moyen Âge] distingue nettement le théoricien (artifex theorice) de celui que nous appelons le créateur (artifex practice). Le premier parle de l'art, le second agit par art. Mais chez l'un comme chez l'autre, la dignité de l'art vient de sa participation à un savoir organisé », E. de BRUYNE, Etudes d'esthétique médiévale, vol. 1, éd. Albin Michel, Paris, 1998 [Bruges, 1946], p. 744.
} 
dans la partie supérieure, d'un tailleur de pierre grimpé dans une tour. L'association de la pratique de l'architecture avec la géométrie n'a rien de surprenant à une période où l'art des proportions, la notion de congruentia, est connue et célébrée par les plus grands maîtres d'œuvre ${ }^{31}$. Si la célébration conjointe des arts libéraux et des arts mécaniques n'est pas une nouveauté en cette fin du $\mathrm{XV}^{\mathrm{e}}$ siècle puisque nous là trouvons dès le début du $\mathrm{XIV}^{\mathrm{e}}$ siècle parmi les vingt-et-un panneaux de marbre de forme hexagonale, initiés par Giotto et réalisés en partie par Andrea Pisano et son atelier à la base du Campanile de Florence (1330-1350) ${ }^{32}$, elle demeure cependant rare et répond à chaque fois à un programme visant à célébrer l'universalité des savoirs.

Une question cependant demeure au sujet de cette pièce, celle de la représentation de l'homme devant son comptoir tenant un livre et comptant à l'aide de jetons. Ce personnage correspond, non pas à l'image de la Géométrie mais celle de l'Arithmétique qui pouvait se trouver sur la partie gauche de la pièce aujourd'hui disparue. La tapisserie est en effet incomplète et devait comprendre plusieurs arts assemblés comme le montre l'ange de droite qui tient dans sa main droite le fragment d'un pan de pavillon et dont le regard est dirigé vers une image aujourd'hui perdue. La tapisserie de Londres présentait donc une composition identique à celle de Rochester. Il s'agissait pour ces tapisseries de rassembler sur une seule pièce plusieurs arts, peut-être dans une démarche assez proche des tentures royales précédemment évoquées.

Le deuxième groupe de tapisseries, produites au début du $\mathrm{XVI}^{\mathrm{e}}$ siècle $-1510-1520$ environ -, renouvelle considérablement la représentation des arts libéraux en en transformant

31 J. ACKERMAN, " "Ars sine scientia nihil est". Gothic Architecture at the Cathedral of Milan », The Art Bulletin, 31 ,1949, p. 84-111.

${ }^{32}$ Associées dans ces œuvres à des épisodes de l'Histoire Sainte, aux prophètes, aux évangélistes, aux vertus cardinales et théologales et aux arts mécaniques, les arts libéraux prennent ici place dans des cycles visant à célébrer l'ampleur et les ambitions intellectuelles et spirituelles de Florence au début du Trecento, voir à ce sujet NORMAN, op. cit. (notre note 19) et VERDIER, op. cit. (notre note 5) p. 324-328. 
le type iconographique stabilisé depuis le XIIe siècle. Il regroupe actuellement quatre pièces : L'Astronomie ${ }^{33}$ (Musée de Göteborg) (Fig. 4), La Rhétorique (Paris, Musée des Arts Décoratifs) (Fig. 5), L’Arithmétique (Paris, Musée national du Moyen Âge) (Fig. 6) et La Musique (Boston, Museum of Fine Arts) (Fig. 7). Les liens qui unissent les trois dernières de ces œuvres à la première ne concernent que le répertoire formel et non le choix iconographique. Nous trouvons en effet dans toutes ces tapisseries un type d'encadrement commun fondé sur la présence de supports sur les bords latéraux (L'Arithmétique, La Rhétorique et L'Astronomie) et d'une limite basse, matérialisée par des végétaux représentés au naturel, sur laquelle brochent les tituli. Ces dispositifs entrainent « un effet barrière » provoquant un éloignement de l'image et un creusement de la surface de représentation. La parenté formelle concerne également les personnages dont les vêtements et coiffures proviennent du même répertoire et se développent selon une même volumétrie. Les plis fortement creusés sont composés d'arêtes aux saillies claires marquant les impacts lumineux. Les visages arrondis des dames aux lourdes paupières, sont cernés de bandeaux à oreilles qui laissent échapper sur l'un des cotés de la tête des voiles soulevés par le vent. Cette parenté formelle a permis de supposer que ces tapisseries furent produites dans un même contexte et par un même groupe de cartonniers ${ }^{34}$.

Cependant, les cartons de ces quatre œuvres ne répondent pas à une commande unique mais à bien deux, la distinction se faisant à partir des choix iconographiques ayant dictés la représentation des arts et de leurs compagnons. L'Astronomie conservée à Göteborg apparaît ainsi comme un unicum en plaçant la figure allégorique non plus trônant mais debout, au sein d'un vaste paysage nocturne dominé par le ciel étoilé. Les marqueurs hiérarchiques

33 E. STRÖMBERG, «"Astronomie", en frank medeltidsgobeläng », Rösska Konstslöjdmuseets, Årsbok 1963-1964, Göteborg, 1965, p. 15-28.

${ }^{34}$ Se reporter principalement à A. S. CAVALLO, Tapestries of Europe and the Colonial Peru in the Museum of Fine Arts, Boston, Boston, 1967, vol. I, p. 77-79; Chefs d'œuvre de la tapisserie du XIV au XVI siècle, Catalogue d'exposition, Grand Palais, 26 octobre 1973-7 janvier 1974, p.155-157 ; F. JOUBERT, La tapisserie médiévale au Musée de Cluny, Paris, 1987, p. 164-165. 
traditionnels (trône, degrés) laissent la place à une simple couronne et au nom porté sur un phylactère qui désignent le personnage principal de l'image. Son geste déictique place l'Astronomie sous l'autorité des astres et plus particulièrement sous celle de la lune qui, accompagnée d'une comète et d'étoiles filantes, est située juste au dessus d'elle. Elle est accompagnée de quatre hommes et dialogue avec l'un d'entre eux. Tenant une sphère armillaire dans la main gauche et un feuillet roulé dans la main droite, ce personnage, bourse au coté et vêtu d'un long manteau, dont les pans s'envolent pour dévoiler une courte robe, à été proposé ${ }^{35}$ comme étant Johannes Müller von Koninsberg autrement connu sous le nom de Regiomontanus $^{36}$, astronome d'origine germanique (1436-1475) auteur d'almanachs et de calendriers imprimés. A ses cotés, assis devant un astrolabe et vêtu de la robe longue des clercs se trouve peut-être, et cette fois suivant la tradition, Ptolémée. Les deux hommes représenteraient ainsi pour les contemporains de l'œuvre la permanence du savoir, du théoricien de l'Antiquité jusqu'au savant contemporain. L'insistance sur la continuité de la pratique de l'Astronomie à travers les âges se retrouve par ailleurs confirmée dans celle des deux bergers dont les Almanachs (ou Calendriers), diffusés grâce à l'imprimerie depuis $1491^{37}$, présentent, sous le topos d'un savoir paysan, des méthodes de lecture et d'interprétation des astres destinés à un public savant.

Le choix fait pour les trois autres œuvres est très éloigné de la tapisserie de Göteborg. La Rhétorique, L'Arithmétique et La Musique forment un groupe iconographiquement cohérent et répondent à des procédés de mises en œuvre communs. En effet, une série de reprises de cartons réunie ces trois tapisseries et les relie à des tentures telles que celle de

\footnotetext{
${ }^{35}$ Chefs d'œuvre de la tapisserie, p. 157.

${ }^{36}$ Sur la carrière de Regiomontanus et ses œuvres se reporter à E. ZINNER, Regiomontanus, His Life and Work, Studies in the History and Philosophy of Mathematics, vol. 1, Amsterdam/New York/Oxford/Tokyo, 1990. La diffusion de l'œuvre de Regiomontanus est importante dans toute l'Europe à la fin du XVe siècle. A titre d'exemple, l'un des utilisateurs des almanachs ou éphémérides de Regiomontanus fut Christophe Colomb au cours de ses voyages vers l'Amérique, ibid., p. 119-125. ${ }^{37}$ La première édition du texte est datée de 1491 chez l'imprimeur parisien Guiot Marchand. M. ENGAMMARE (dir.) Calendrier des bergers, PUF, collection Sources, Paris, 2008.
} 
l'Histoire de l'enfant prodigue ou des Scènes de Chasse du Musée National du Moyen Âge comme à des gravures germaniques du début du XVI siècle. C'est ainsi le cas pour l'homme de profil dont la coiffure est composée d'un bonnet plissé, laissant apparente l'oreille, et d'un chapeau aux bords échancrés rabattu sur la surface de la représentation parachevé d'un plumet. Le prototype, qui se retrouve dans une gravure de Lucas van Leyden datée de $1508^{38}$, est repris à la fois dans les pièces de L'Arithmétique, de La Musique et celle de L'Enfant prodigue (Fig. 8). Le motif de l'homme au large manteau tourné de trois-quarts dos est commun à L'Arithmétique et à L'Enfant prodigue (Fig. 9). Un recours direct à la gravure se remarque dans le motif du joueur de flute traversière, dont le motif original se trouve chez le maître MZ autour de 1500, repris à la fois dans la tapisserie de Boston et dans la tapisserie des Scènes de chasse (Fig. 10) Enfin, si l'enfant nu sonnant de la trompette renvoie à des séries de putti très largement diffusé en gravure à la fin du $\mathrm{XV}^{\mathrm{e}}$ siècle ${ }^{39}$ sans qu'il nous ait été possible d'en identifier le prototype, le chien à la crinière de lion représenté au pied de Dame Rhétorique est la citation parfaite de celui assistant à la Visitation dans une gravure de Dürer des années 1504 (Fig.11) et celui plus efflanqué de la tapisserie de L'Arithmétique dérive du même corpus sans en être cependant une citation fidèle.

Ces processus d'élaboration, fondés sur la reprise de cartons préexistants ou de gravures certainement présentes dans les ateliers, a conduit la critique à considérer ces œuvres comme des images produites à moindre coût et donc sans réel intérêt iconographique ${ }^{40}$. Les postures des personnages (l'homme se retournant dans L'Arithmétique par exemple) comme leurs attributs ont été interprétés comme répondant aux exigences de la production et non pas à un programme iconographique pensé et élaboré en dehors de l'atelier des lissiers. Cependant, ces œuvres, dont nous ne connaissons aucun retissage et dont le thème est

\footnotetext{
${ }^{38}$ Saint Georges, c. 1508,164 x 121.

${ }^{39}$ Ce type de putti se retrouve à la fois chez Albrecht Dürer et Daniel Hopfer au tournant du XVI ${ }^{\mathrm{e}}$ siècle.

${ }^{40}$ F. JOUBERT, op. cit. (notre note 34) p. 164-165.
} 
particulièrement rare en tapisserie, est ici présenté suivant des orientations iconographiques complexes et novatrices.

Dans ces trois œuvres, la personnification féminine de l'Art se tenait à l'origine au centre de la composition comme l'indiquent à la fois les deux tituli conservés et les personnages désormais coupés par les limites latérales. Les femmes sont maintenues, pour deux d'entre elles, sur un trône et si l'Arithmétique se tient debout, elle demeure associée à un dais d'honneur remplissant les mêmes fonctions hiérarchiques que les sièges. Aucun savant ni philosophe n'apparait désormais à leur coté. Entourées exclusivement de compagnons masculins, elles semblent orchestrer la pratique de leur art, comme le rappelle l'inscription désormais en français portée au dessus de leur tête («Dame Rethoricque »; « Aritmetique »; « Musicque »).

Si le choix de représenter la pratique de l'art et non plus ses seuls théoriciens bouleverse radicalement le prototype primitif, il ne renvoie cependant pas à une iconographie commune qui se résumerait à l'accumulation d'outils ou de praticiens dans le simple usage de leur métier. Ainsi, la tapisserie de La Musique représente un ensemble de musiciens qui convergent vers la personnification féminine de leur art. Assise sur un trône architecturé, qui rappelle ceux associés à la Vierge dans les œuvres italiennes au cours du $\mathrm{XV}^{\mathrm{e}}$ siècle tant en peinture qu'en gravure, la Musique joue du positif (petit orgue portatif) dont les soufflets sont actionnés par un assistant tout en suivant de sa main droite la partition déroulée sur son accoudoir. Le choix de cet instrument polyphonique dont les sonorités sont réputées pour imiter les «modulations de la voie humaine ${ }^{41}$, renvoie à la place qu'il tient dans l'instrumentarium médiéval. Investi d'un symbolisme mystique par les Pères de l'Eglise, l'orgue, instrument des chapelles et des cathédrales, nécessite dans « son jeu une connaissance

\footnotetext{
${ }^{41}$ M. CLOUZOT, Images de musiciens (1350-1500). Typologie, figuration et pratiques sociales,
} Turnhout/Tours, 2007, p. 246. 
approfondie de la musique théorique ${ }^{42}$ » et fait écho, dans cette image, à la fonction même de la personnification. Il en est également ainsi pour le dulcimer duquel l'homme assis à ses pieds joue, en le frappant à l'aide de plectres. Dérivé de la famille du psaltérion, le dulcimer, est symboliquement associé à la pédagogie et à la théorie musicale comme les autres instruments à cordes pincées représentées. Luth, rebec et harpe accompagnent le chanteur et illustrent autant la pratique musicale que le «savoir spéculatif et mathématique de la musica $^{43} \gg$. Tous ses instruments de bas, aux sons faibles et doux, sont représentés dans le premier cercle entourant l'allégorie de la Musique. Un peu plus loin sont ajoutés des instruments de hauts, flutes, tambour et cornemuses qui évoquent une pratique plus populaire et plus sonore de l'art musical. Le fou revêtu de son coqueluchon et de son habit rayé aux jambes ornés de grelots joue de la cornemuse et marque à la fois le point le plus éloigné et le plus bruyant de la composition. La représentation de 1'art de la Musique est donc à la fois celle de la pratique musicale, suivant une déclinaison très large puisqu'elle évoque à la fois la musique sacrée à travers le positif jusqu'à la plus populaire comme celle du tambour et de la cornemuse, et celle de sa dimension théorique aux travers de la figuration d'instruments rappelant ses fonctions spéculative et théologique. Cette iconographie savante est soutenue par le titulus en latin rappelant la fonction théorique de l'ars musica et la place qu'elle tient parmi les arts du quadrivium universitaire : «Invenere locum per me modulamina vocum. Dat notula scire musica docta lire $»^{44}$.

La représentation de l'Arithmétique répond à une logique identique en mettant en scène l'application pratique de son processus théorique. L'allégorie féminine se tient devant un comptoir où sont disposés un ensemble de jetons métalliques sortis d'une boite ouverte posée à son angle gauche. A cette évocation de la numération et du calcul dérivés de l'abaque

\footnotetext{
${ }^{42}$ CLOUZOT, p. 246

${ }^{43}$ Idem, p. 248.

44 «La mesure des voix par moi a trouvé sa place. La musique apprise sur la lyre donne la connaissance des notes. »
} 
antique sont associés les chiffres arabes portés sur le livre ouvert que l'homme assis tient incliné. Cette présentation des deux procédés de numération accompagnant l'image de l'Arithmétique renvoie à l'illustration d'ouvrages didactiques tels que la Margarita Philosophica de Gregor Reisch publié pour la première fois en 1503, dont l'allégorie féminine du Typus Arithmeticae domine Boèce et Pythagore occupés au calcul algorithmique à partir de chiffres tracés à même une table et d'un abaque et de ses jetons. La cohabitation entre algoriste et abaciste dans cette image, reprise dans la tapisserie de Cluny, témoigne de la pratique contemporaine de ces deux systèmes et de leur intégration depuis le XIII ${ }^{\mathrm{e}}$ siècle à l'enseignement universitaire ${ }^{45}$. La particularité de la tapisserie est cependant de combiner cette mise en image des deux systèmes arithmétiques alors existant avec l'évocation de leur application dans le monde fiscal et commercial. Si le choix de représenter un comptoir et non pas un abaque avec ses lignes rigoureusement tracées invite à cette lecture et renvoie au monde des marchands, des banquiers et des officiers de la finance ${ }^{46}$, elle est plus particulièrement renforcée par la représentation d'un instrument de crédit tout à fait particulier. L'homme qui se tient debout à droite porte dans sa main un petit arc dont la corde retient une série de baguettes de bois de longueurs différentes. Il s'agit là de ce que Godefroy ${ }^{47}$ répertorie comme des taillettes (tailleite) c'est-à-dire des petits morceaux de bois sur lesquels sont pratiqués des encoches (des tailles) destiné à enregistrer, dans le monde du commerce, un crédit. Cet outil, dont l'utilisation est attestée jusqu'à la fin du XX $\mathrm{X}^{\mathrm{e}}$ siècle tant

\footnotetext{
45 M. PASTOUREAU, Jetons, méreaux et médailles, Typologie des sources du Moyen Âge occidental, fasc. 42, Turnhout, 1984.

46 «Nombreux sont ceux qui sont conduits, par leur charge ou par leur profession, à utiliser des jetons : marchands, artisans, changeurs, banquiers et tout leur personnel ; mais aussi officiers et commissaires financiers, fiscaux, monétaires (élus, receveurs, payeurs, trésoriers, collecteurs, contrôleurs, vérificateurs, etc.) ; ainsi que les «clercs » de tous rangs employés aux comptes dans les hôtels royaux, princiers ou seigneuriaux dans les villes, dans les communautés religieuses, dans les chambres des comptes, les cours des aides et les administrations de toutes natures. », ibid., p. 19

${ }^{47}$ F. GODEFROY, Dictionnaire de l'ancienne langue française et de tous ses dialectes, du IX au XV siècle, composé d'après le dépouillement de tous les plus importants documents manuscrits ou imprimés qui se trouvent dans les grandes bibliothèques de la France et de l'Europe, Paris, 18801902, vol. 7.
} 
dans les provinces reculées de Hollande que dans les campagnes françaises ${ }^{48}$, et dont cette image semble constituer un unicum, oriente résolument le thème de cette tapisserie vers la représentation de l'application de l'arithmétique dans le monde du commerce et de l'usure. C'est également à cela que renvoie l'homme situé sur la gauche et dont la bourse est exposée avec ostentation.

Cette tapisserie qui peut être lue comme une célébration du commerce et de la richesse qu'apporte la mise en œuvre de l'art de l'arithmétique trouve un second niveau de signification par la présence de deux motifs aux accents teintés de morale chrétienne. Parmi les médailles que quatre des sept hommes représentés portent sur leur chapeau ${ }^{49}$, celle de l'homme tenant le livre se distingue car elle porte le monogramme du Christ (IHS). Ce personnage qui pointe de son doigt le contenu du texte qui semble guider l'action de l'Arithmétique introduit donc dans l'œuvre une portée morale. La présence du Christ sous une de ses formes symboliques en plein cœur de la représentation place la pratique du commerce sous l'autorité de la pensée chrétienne et permet également de maintenir dans cette œuvre le lien traditionnel des arts libéraux avec l'Eglise. Peut-être est-ce dans ce contexte que doit être replacé le personnage de droite qui se détourne de la scène tout en tenant dans son dos un sac vide à l'ouverture béante. Ce personnage se retrouve à l'identique, mais inversé, comme il a été dit précédemment dans la tapisserie de l'Enfant prodigue du Musée National du Moyen

\footnotetext{
${ }^{48}$ D. BURGER, op. cit. (notre note 15) p. 861, mentionne l'usage de cet instrument de crédit à Oostburg dans le sud des Pays-Bas ou sur l'Ile d'Amaland dans les années 1950. Ce même type d'outil était encore utilisé dans certaines régions françaises comme l'Agenais dans les années 1980 dans le commerce itinérant (je remercie M. Gil pour cette information). La langue allemande conserve également le souvenir de cette pratique : "La taillette se dit "Kerbholz" en allemand (holz = bois, kerbe $=$ entaille), et l'expression "etwas auf dem Kerbholz haben" (avoir quelque chose sur la taillette) est encore courante dans l'allemand contemporain. Cela se dit d'une personne qui a un passé louche, un casier judiciaire, etc... C'est donc une expression qui n'a conservé que son sens figuré, avec une connotation pénale forte, et le sens matériel s'est perdu. » Je remercie Julia Drobinsky avec ces précisions d'avoir complété l'explication du terme taillette .

${ }^{49}$ Le motif de la médaille sur les chapeaux se retrouve également dans les tentures de L'Enfant prodigue, des Scènes de chasse (Paris, Musée National du Moyen Âge), et de Judith et Holopherne (Bruxelles, Musées Royaux d'Art et d'Histoire), JOUBERT, op. cit. (notre note 31), p. 160. Cependant aucune d'entre elles ne semblent présenter le monogramme IHS.
} 
Âge. Si sa fonction plastique est de faire formellement écho à l'homme à la bourse au coté qui se trouve sur la gauche de l'image, son sens iconographique est peut-être également lié à ce même personnage. Sa posture, la simplicité de son vêtement comme le sac vide sont les attributs antithétiques de ceux revêtus par l'homme à la bourse pleine. Ce motif de remploi n'a donc peut-être pas été choisi au hasard mais comme contrepoint à l'image de la fortune et de l'opulence ${ }^{50}$.

La tapisserie de la Rhétorique met en scène, comme les pièces précédemment étudiées la personnification féminine de l'art accompagnée de ses praticiens. Définie par Quintillien comme ars bene dicendi ${ }^{51}$, la Rhétorique se décline à la période médiévale selon trois modes de pratique exposés ici dans la tapisserie : l'art de prêcher (artes praedicandi), l'art d'écrire (artes dictaminis) et les arts poétiques (artes poetriae) $)^{52}$. Livres et rouleaux sont présentés dans une petite pièce latérale alors que l'assemblée masculine placée sous l'autorité de «Dame Réthoricque » écoute l'un de ses membres déclamer son texte. Vêtus de robes ou de longs manteaux et coiffés de chapeaux plats, les hommes semblent se soumettre au jugement de leur art dont la personnification brandit deux couronnes, l'une d'entre elle étant fichée en haut d'un bâton. Seule tapisserie où l'allégorie féminine porte une titulature renvoyant au monde romanesque de la fin du Moyen $\hat{A}_{g} \mathrm{e}^{53}$ faisant écho au sens même de l'art représenté, cette œuvre développe une thématique commune à celles de Cluny et de Boston. Développant une iconographie savante des arts et de leurs pratiques, ces pièces proposent aux

\footnotetext{
${ }^{50}$ Le choix de ce personnage est peut-être également à mettre en lien avec son utilisation dans la tapisserie de l'Enfant prodigue. Si l'on suit G. SOUCHAL (Chefs d'œuvres du Musée de Cluny. Les tapisseries médiévales. (Publications filmées d'Art et d'Histoire), Montrouge, 1959, p. 51) dans son hypothèse d'y reconnaître le fils aîné jaloux du sort favorable réservé à son frère cadet dans la parabole de l'Evangile de Luc (15. 11-13), ce personnage est dans les deux cas associé au dépouillement même si le sens de lecture est différent dans ces deux œuvres.

${ }^{51}$ QUINTILLIEN, Inst. II, 17, 37 et ISIDORE, Et. II, 1,1 « Rhetorica est bene dicendi scientia in ciuilibus quaestionibus eloquentia copia ad persuadendum iusta et bona ».

${ }^{52}$ R. MEYENBERG, Alain Chartier prosateur et l'art de la parole au XV siècle. Etudes Littéraires et Rhétoriques. Romanica Helvetica vol. 107, Berne, 1992, p. 58.

${ }^{53}$ A. STRUBEL, "Grand senefiance $a$ » Allégorie et littérature au Moyen Âge, Paris, 2009, p. 321323
} 
« regardeurs » une version nouvelle des arts libéraux puisque libérée de l'autorité des figures savantes, qui leur étaient associées depuis leurs origines littéraire et figurative, au profit de leurs praticiens qui acquièrent ici un statut remarquable. L'iconographie choisie ouvre peutêtre vers de nouveaux types de commandes et d'exposition en éloignant les arts de leurs fondements antiques et donc de leur classification universitaire. Arithmétique, Musique ou Rhétorique, désormais montrées dans leur pratique les plus savantes (jetons, dulcimer) comme les plus populaires (cornemuse, taillettes), et non plus uniquement reliées au vaste ensemble des disciplines du savoir universel, peuvent être commandées pour elles-mêmes et exposées seules tout en faisant sens pour leur commanditaire et leur public. Ces œuvres montrent également une pratique des remplois de cartons et de modèles gravés tout à fait particulier dans le sens où les figures remployées sont investies de fonctions narrative ou symbolique précises et ne sont pas considérées comme de simples silhouettes vides de sens. La recomposition des motifs est donc ici exploitée suivant un programme iconographique précis n'ayant pas été laissé à l'initiative du seul chef d'atelier. La commande est sans nul doute dirigée et l'exercice des remplois relève de l'imbrication étroite des tâches de l'iconographe et du concepteur des cartons ${ }^{54}$.

Si des représentations sérielles des arts libéraux ont sans nul doute existé comme nous le rappelle les rares mentions d'archives et les pièces de Londres et Rochester, les tapisseries du début du $\mathrm{XVI}^{\mathrm{e}}$ siècle semble répondre à d'autres choix rompant avec la tradition qui perdure à la même époque en peinture et en sculpture ${ }^{55}$. La présence répétée des praticiens au coté de la personnification de leur art renvoie à des formules iconographiques présentes dans la gravure au début du XVI ${ }^{\mathrm{e}}$ siècle à l'instar de l'ouvrage de Gregor Reisch déjà cité. Les choix iconographiques rejoignent, semble t-il ici, les pratiques d'atelier qui font alors la part

\footnotetext{
${ }^{54}$ Catheline Périer d'Ieteren y voit la main d'un peintre anversois (Compte rendu de JOUBERT, op. cit. (notre note 31) dans Revue Belge d'Archéologie et d'Histoire de l'art, ${ }^{\circ} 75,1988$, p. 120).

${ }^{55}$ Se reporter aux exemples cités par VERDIER, op. cit. (notre note 5).
} 
belle aux modèles gravés. La conception des œuvres doit dés lors être comprise comme un processus globalisé où formes plastiques et sens iconographiques sont puisés aux mêmes sources sans pour autant en être des répliques serviles. La rareté constatée de la représentation des Arts libéraux en tapisserie a semble t-il ouvert sur des commandes dirigées ayant permis de s'éloigner de la tradition iconographique et plus encore du texte fondateur de Martianus Capella. 


\section{Liste des figures et légendes des images :}

1. L'Arithmétique et L'Astronomie, Rochester, Memorial Art Gallery (H. 300 x L. 270 cm.).

2. La Géométrie, Londres, Museum of Freemasonry (H. 292 x L. 163 cm).

3. Les Arts libéraux : la Grammaire et Priscien, la Logique et Aristote, la Rhétorique et Cicéron, la Musique et Tubal, Le Puy-en-Velay, Cathédrale, Peinture murale de l'ancienne bibliothèque, Relevé de Louis Joseph Yperman, 1893, Médiathèque de l'Architecture et du Patrimoine (Saint-Cyr,1996/089/)

4. L’Astronomie, Göteborg, Röhsska Konstslöjdmuseet (H. 240 x L. 340 cm)

5. La Rhétorique, Paris, Musée des Arts Décoratifs (H. 2.55 x L. 2.90 m.)

6. L'Arithmétique, Paris, Musée national du Moyen Âge (Musée de Cluny), (H. 315 x L. 294 cm.).

7. La Musique, Boston, Museum of Fine Arts (H. 322 x L. 337 cm).

8. Tapisserie de l'enfant prodigue, Musée national du Moyen Âge (Musée de Cluny), détail inversé ; L’Arithmétique, Paris, Musée national du Moyen Âge (Musée de Cluny), détail ; La Musique, Boston, Museum of Fine Arts, détail ; Lucas van Leyden, Saint Georges, c. 1508, détail inversé

9. L’Arithmétique, Paris, Musée national du Moyen Âge (Musée de Cluny), détail ; Tapisserie de l'enfant prodigue, Musée national du Moyen Âge (Musée de Cluny), détail inversé.

10. La Musique, Boston, Museum of Fine Arts, détail ; Scènes de chasse, Paris, Musée national du Moyen Âge (Musée de Cluny), détail ; Maître M.Z., Les soldats, c. 1500, Paris, BnF Cabinet des estampes, détail.

11. Albrecht Dürer, Vie de la Vierge : 8. La Visitation, 1503, Gravure sur bois, Munich, Staatliche Graphische Sammlung, détail. 


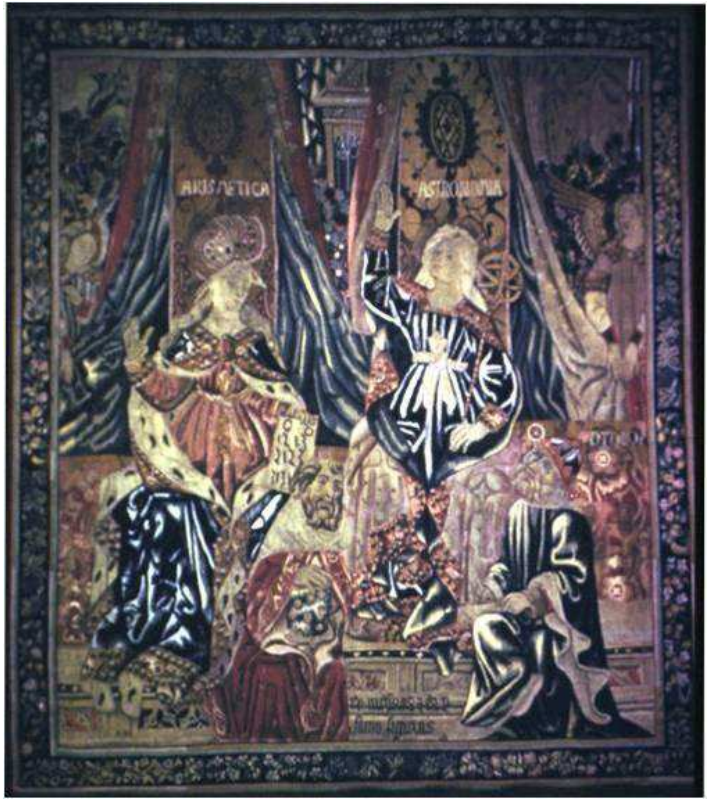

1. L'Arithmétique et L'Astronomie, Rochester, Memorial Art Gallery (H. $300 \mathrm{x}$ L. $270 \mathrm{~cm}$.).

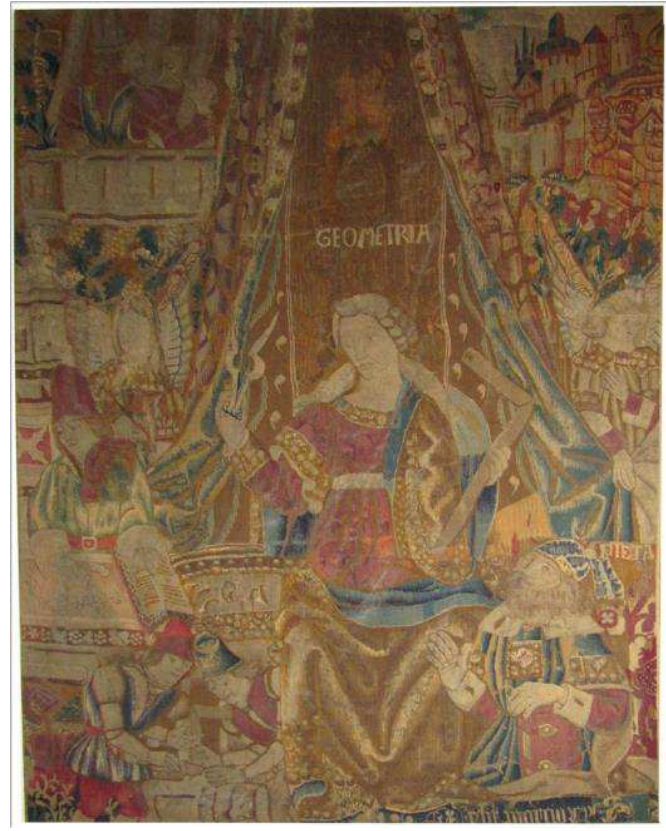

2. La Géométrie, Londres, Museum of Freemasonry (H. 292 x L. 163 cm). 


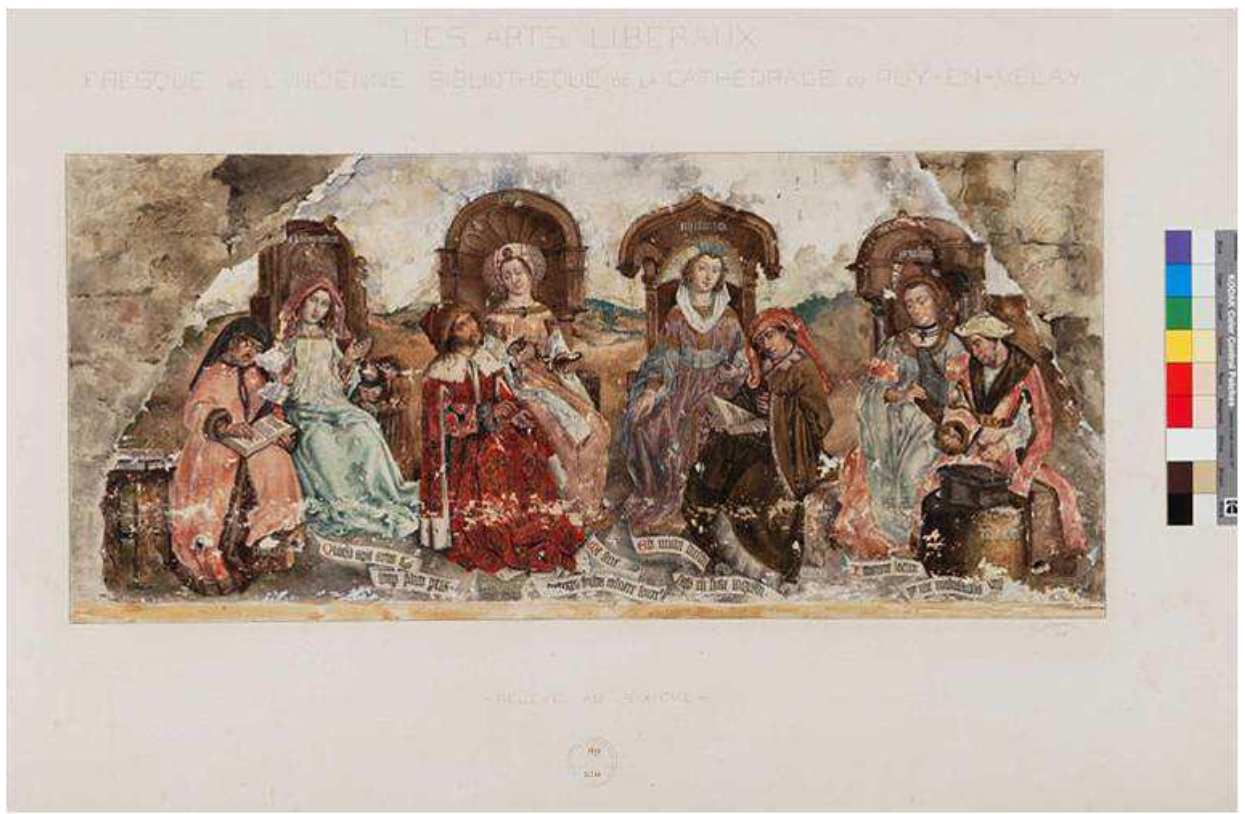

3. Les Arts libéraux : la Grammaire et Priscien, la Logique et Aristote, la Rhétorique et Cicéron, la Musique et Tubal, Le Puy-en-Velay, Cathédrale, Peinture murale de l'ancienne bibliothèque, Relevé de Louis Joseph Yperman, 1893, Médiathèque de l'Architecture et du Patrimoine (Saint-Cyr,1996/089/)

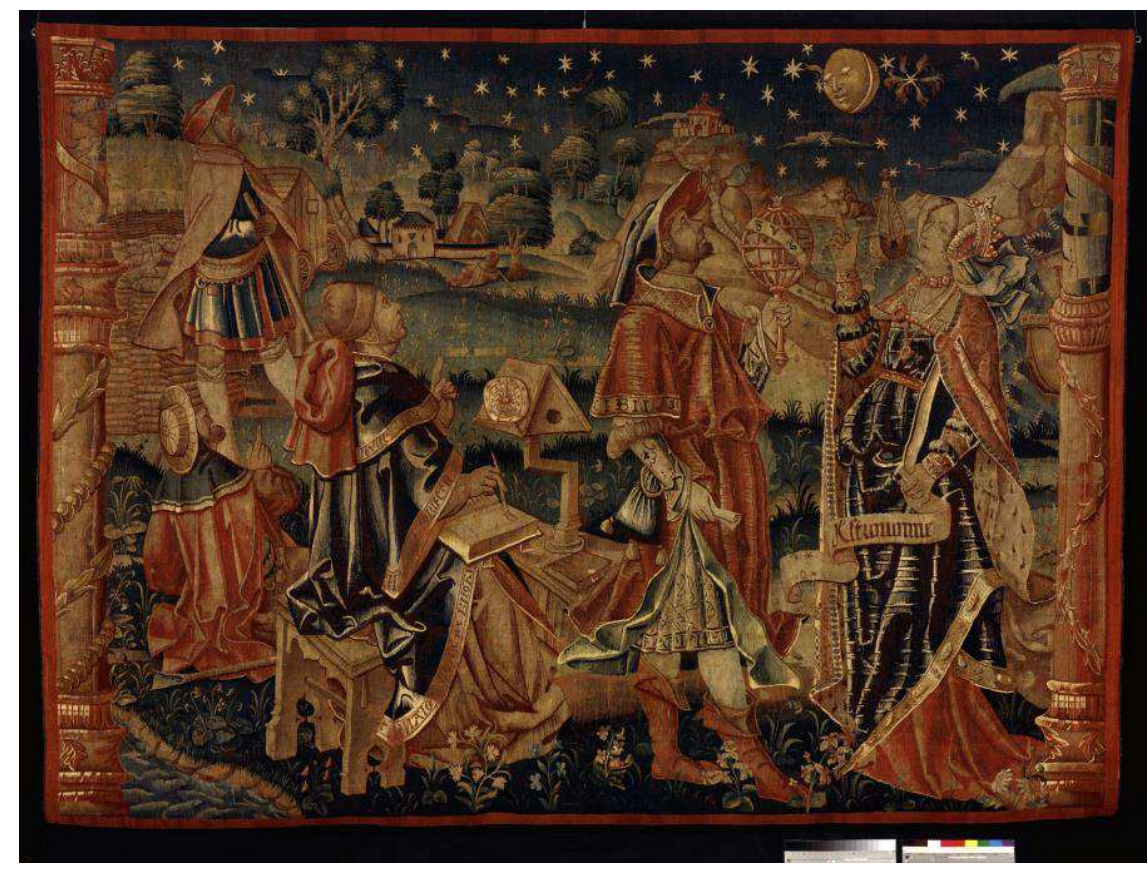

4. L'Astronomie, Göteborg, Röhsska Konstslöjdmuseet (H. 240 x L. 340 cm) 


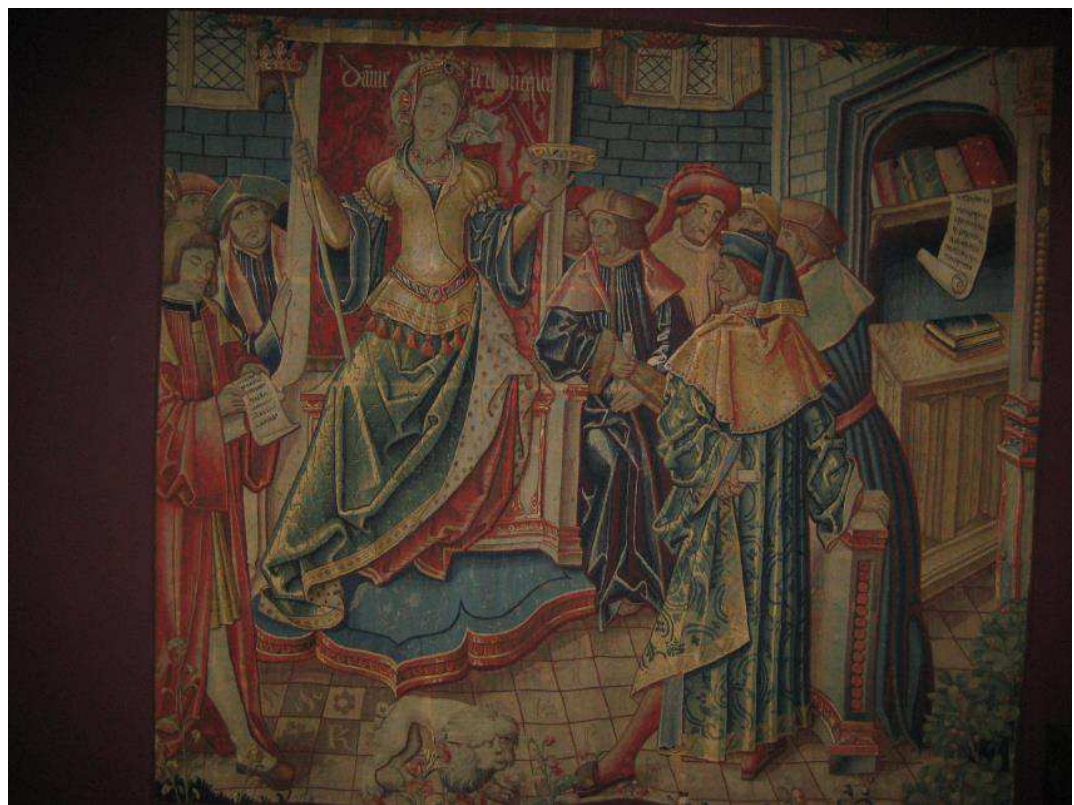

5. La Rhétorique, Paris, Musée des Arts Décoratifs (H. 2.55 x L. 2.90 m.)

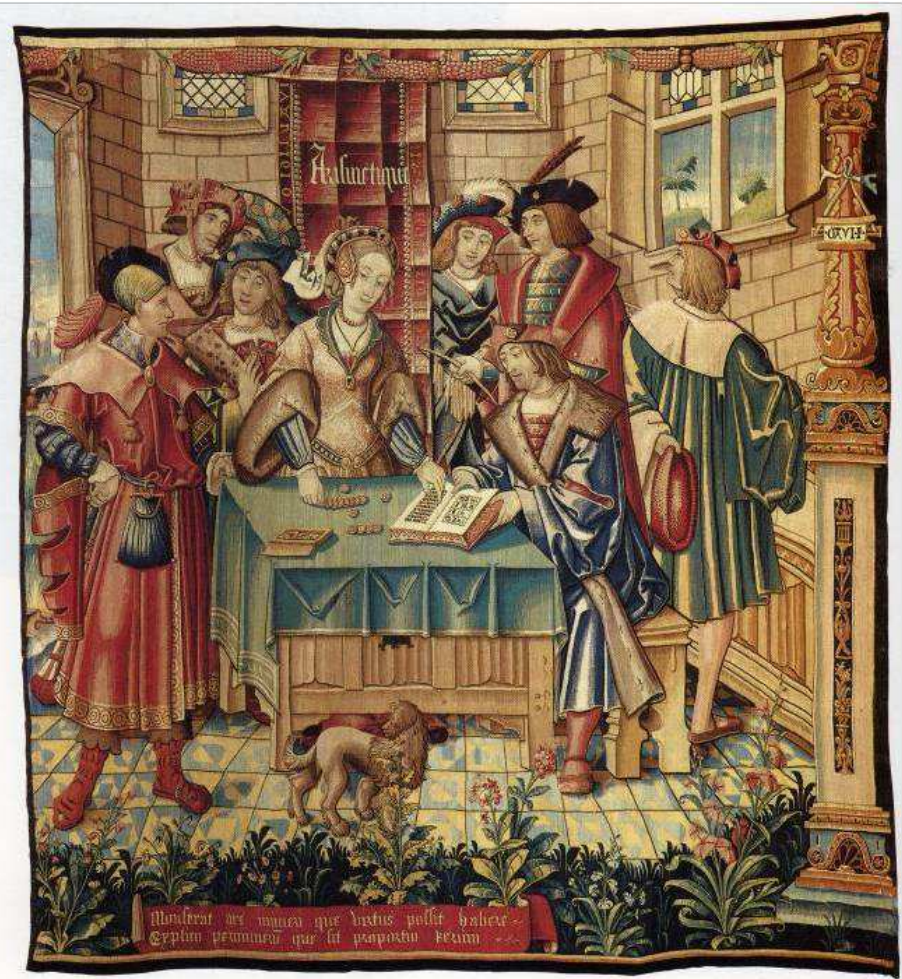

6. L'Arithmétique, Paris, Musée national du Moyen Âge (Musée de Cluny), (H. 315 x L. 294 cm.). 


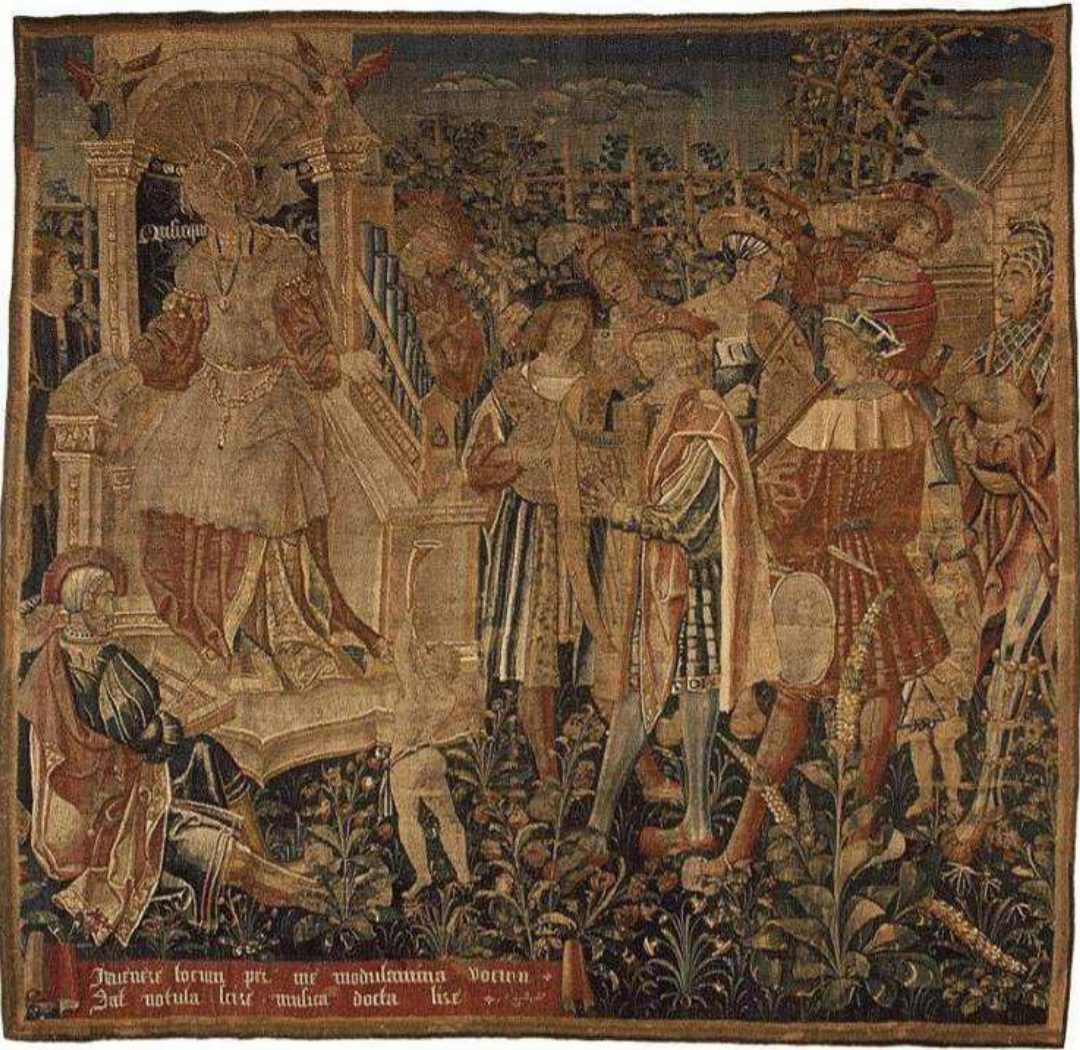

7. La Musique, Boston, Museum of Fine Arts (H. 322 x L. 337 cm).
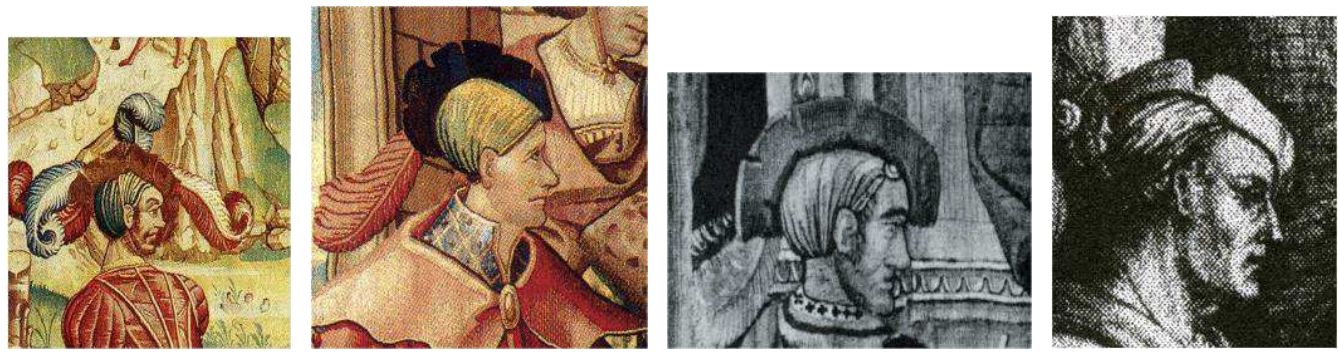

8. Tapisserie de l'enfant prodigue, Musée national du Moyen Âge (Musée de Cluny), détail inversé ; L'Arithmétique, Paris, Musée national du Moyen Âge (Musée de Cluny), détail ; La Musique, Boston, Museum of Fine Arts, détail ; Lucas van Leyden, Saint Georges, c. 1508, détail inversé. 


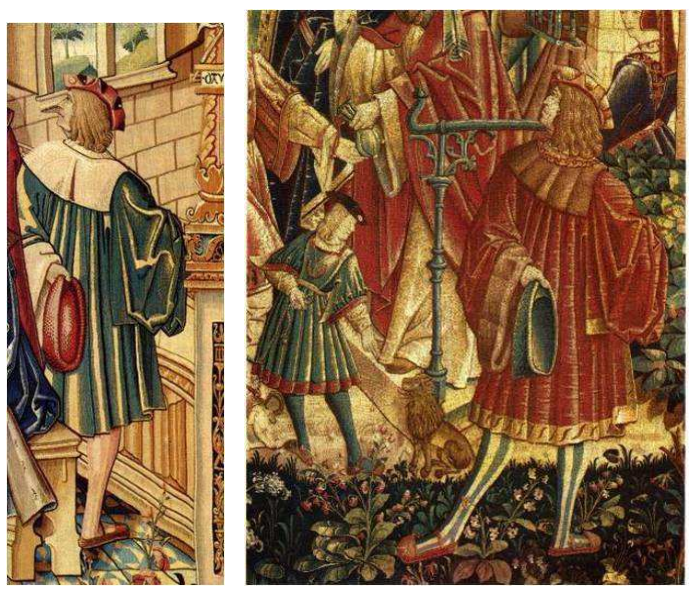

9. L'Arithmétique, Paris, Musée national du Moyen Âge (Musée de Cluny), détail ; Tapisserie de l'enfant prodigue, Musée national du Moyen Âge (Musée de Cluny), détail inversé.
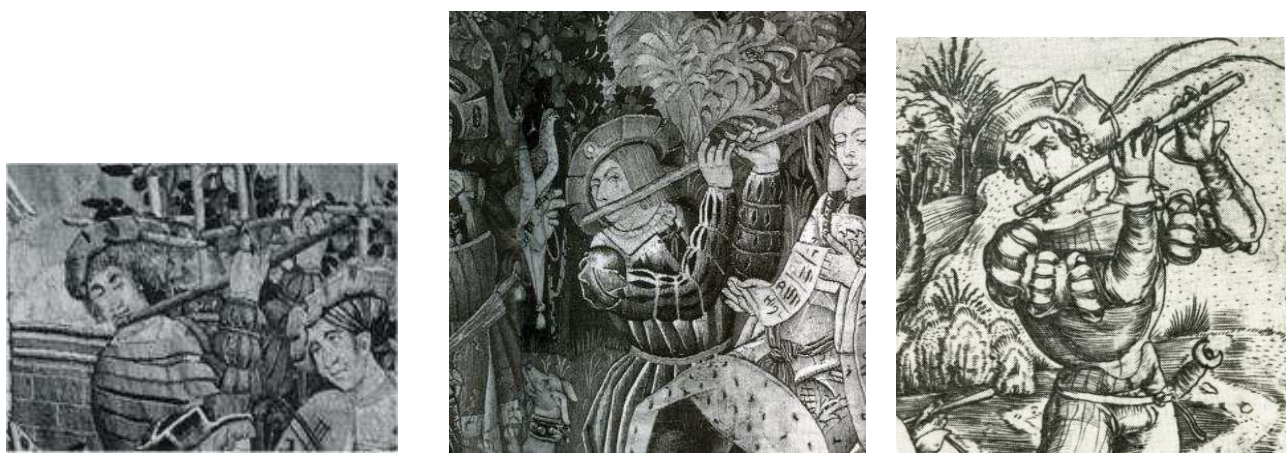

10. La Musique, Boston, Museum of Fine Arts, détail ; Scènes de chasse, Paris, Musée national du Moyen Âge (Musée de Cluny), détail ; Maître M.Z., Les soldats, c. 1500, Paris, BnF Cabinet des estampes, détail.

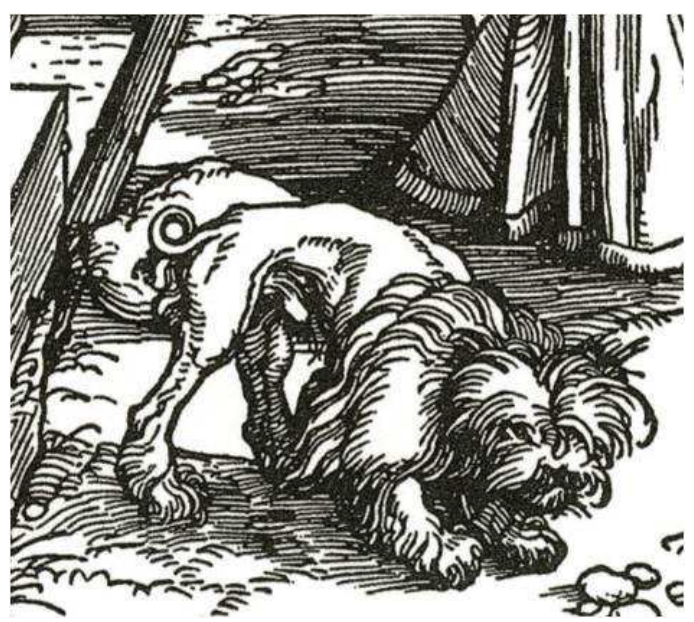


11 Albrecht Dürer, Vie de la Vierge : 8. La Visitation, 1503, Gravure sur bois, Munich, Staatliche Graphische Sammlung, détail. 